Case Study

\title{
Is blood flow-restricted training effective for rehabilitation of a pianist with residual neurological symptoms in the upper limbs? A case study
}

\author{
Tomohiro Yasuda, $\mathrm{PhD}^{1,2)^{*}}$, Yumi Sato ${ }^{3)}$, Toshiaki NaKajima, MD, $\mathrm{PhD}^{2)}$ \\ 1) School of Nursing, Seirei Christopher University: 3453 Mikatahara, Kita-ku, Hamamatsu, Shizuoka \\ 433-8558, Japan \\ 2) Department of Cardiovascular Medicine, School of Medicine, Dokkyo Medical University, Japan \\ 3) Okazaki Women's University, Japan
}

\begin{abstract}
Purpose] We investigated whether blood flow-restricted training known as KAATSU training, was effective for rehabilitation of a pianist with residual neurological symptoms in the upper limbs. [Participant and Methods] A pianist with residual neurological symptoms in the upper body played "Revolutionary Etude" under two conditions: piano performance with (Piano-blood flow-restricted) and without (Piano-control) the restriction of blood flow to the upper limbs. In the Piano-blood flow-restricted exercise, a pressure of 130-170 mmHg was applied around the most proximal portion of both arms. The changes in upper limb circumference and muscle strength were measured before, immediately after, and $15 \mathrm{~min}$ after the performance. The impression of the piano performance was recorded after the Piano-blood flow-restricted exercise. [Results] Immediately after the piano performance, the forearm and upper arm circumferences had increased significantly in both arms, and the change was greater in the Piano-blood flow-restricted than in the Piano-control condition. The handgrip strength for the right arm also showed greater changes in the former than the latter. However, there were no significant differences between the two conditions regarding the handgrip strength of the left arm. [Conclusion] There is a high possibility that blood flow-restricted training is effective for rehabilitation of the pianist with residual neurological symptoms in the upper limbs.

Key words: KAATSU-rehabilitation, Piano performance, Venous blood-flow restricted training
\end{abstract}

(This article was submitted Mar. 7, 2021, and was accepted May 1, 2021)

\section{INTRODUCTION}

According to the Tokyo Metropolitan Police Department, there were 381,237 traffic accidents, resulting in 461,775 injuries in 2020 in Japan ${ }^{1)}$. Moreover, among the fatalities and injuries caused by the traffic accidents, only $0.7 \%$ died within 30 days after the accident while $9.0 \%$ of the victims had residual disabilities. The most commonly injured areas were the neck (30.7\%), the upper limbs (18.6\%), and the head and face (18.4\%). The local neurological symptoms due to brain, neck, and upper limb injuries ${ }^{2}$ persist throughout the life time of the affected individuals and affect their ability to perform daily activities (physical exercise, etc.).

In general, traditional high-intensity exercise training ( $\geq 70 \%$ 1-repetition maximum: $1 \mathrm{RM})$ improves skeletal muscle morphology and function ${ }^{3)}$; however, this method seems is not feasible and potentially dangerous for patients. In contrast, moderate blood flow-restricted (BFR) training (up to 30\% 1RM), known as KAATSU training, can cause muscle hypertrophy

*Corresponding author. Tomohiro Yasuda (E-mail: tomohiro-y@seirei.ac.jp)

(C2021 The Society of Physical Therapy Science. Published by IPEC Inc.

(c) (1) $\odot$ This is an open-access article distributed under the terms of the Creative Commons Attribution Non-Commercial No DerivacC. 
and does not decrease vascular function ${ }^{4,5}$. BFR training is provided with an appropriately pressurized special pressure belt placed around the base of the limbs, which restricts blood flow ${ }^{6}$. Unlike hemostasis using a tourniquet, which completely halts blood flow in both arteries and veins, BFR training is performed by maintaining a restricted blood flow with a specially designed belt, which is carried out while pooling the blood in the upper or lower limbs. Previous studies have revealed that BFR training could improve neuromuscular adaptations with various types of load resistance (e.g., machine, elastic band, electrical stimulation, and body weight $)^{5,7-11)}$. It has been proven that different physical exercises can also improve the neuromuscular mechanisms of the body ${ }^{10,11)}$.

In 2006 and 2016, a nationwide questionnaire survey of BFR training was conducted by researchers in the medical and health sports fields ${ }^{12,13)}$. According to these surveys, BFR training is widely used in various medical and sports settings (105 facilities in 2006 and 232 facilities in 2016), and there have been no reports of any side effects or serious complications. In addition, the questionnaire survey in 2016 reported that BFR training is also widely used in the rehabilitation of patients suffering from various diseases including neuromuscular disease ${ }^{13)}$. Thus, we hypothesized that even in patients with disabilities who have local neurological symptoms, the combined use of BFR exercise with physical exercises the participant is accustomed to on a daily basis provides beneficial effects on neuromuscular mechanisms (muscle hypertrophy and increase in muscle strength, etc.).

Previous studies demonstrated that increases in muscle size may be partly a result of the acute muscle cell swelling observed during and after BFR exercise ${ }^{14,15)}$. Additionally, a previous study reported that increased circumference, an index of muscle cell swelling, was more pronounced in BFR than in non-BFR immediately after exercise ${ }^{16-18)}$. Thus, the purpose of this study was to examine the effects of this combination on maximum muscle strength and circumference of a participant with neuropathy due to the traffic accident after playing the piano with blood flow restriction.

\section{PARTICIPANT AND METHODS}

The participant was a 43 year-old woman (standing height, $157 \mathrm{~cm}$; body mass index, $21.3 \mathrm{~kg} / \mathrm{m}^{2}$; average finger length, left 22.5 and right $23.0 \mathrm{~cm}^{19)}$ ) who presented with local neurological symptoms in the back of the neck and upper limbs due to two previous traffic accidents. Residual disability was certified as 14th grade 9th class in Japan. She was a professional pianist with approximately 39 years of experience and had won first place in the Japan Mozart Music Competition. She had not participated in any resistance-type physical training for a minimum of 5 years prior to the beginning of this study. The participant provided an informed consent after the risks associated with the training and measurements and the purpose of the study, which conformed to the Declaration of Helsinki and was approved by the Ethics Committee for Human Experiments, Dokkyo Medical University Hospital (approval number: 27074) was explained to her.

A grand piano (YAMAHA C3, Yamaha Music Japan Co., Ltd., Hamamatsu, Japan) located in a university's music room (length and width: approximately $11 \mathrm{~m}$ and $6.5 \mathrm{~m}$, respectively) was used. The Chopin "Revolutionary Etude" (Op. 10 No. 12; Performance time, approximately 3 minutes) was selected for the piano performance, which was performed with (PianoBFR) and without BFR (Piano-Ctrl) (Fig. 1). In a randomized order, Piano-BFR and Piano-Ctrl were performed six times each over multiple days. The average of six performances under each condition was represented as a single data point for statistical analysis. The protocols are summarized in Fig. 2.

Before the experiment, the participant was familiarized with a specialized pressure cuff (30 mm width, KAATSU Master, KAATSU JAPAN Co., Ltd., Tokyo, Japan) around the most proximal portion of both arms. The pressure was set to 130-170 $\mathrm{mmHg}$, as described in previous studies $\left.{ }^{17}, 18,20,21\right)$ reported that this was the optimal level of BFR for altering blood flow and energy supply and increasing muscle activation without affecting total work in the upper arm. The restriction pressure was applied to the upper arms, as previously described ${ }^{17,18,20,21)}$. During acclimatization, no signs of discomfort or pain were observed. In the Piano-BFR experiment, the participant was seated on a chair and the cuffs were tightened around the arms to a belt pressure (the pressure the cuff applied to the arm prior to inflation) of $30 \mathrm{mmHg}$ on the most proximal region of the testing arm. The cuffs were then inflated to $130-170 \mathrm{mmHg}$ and were quickly removed immediately after the piano performance. The amount of time under moderate blood flow restriction was approximately 4-5 min.

Before the piano performance session, the resting blood pressure in the sitting position was measured using the oscillometric method. A manchette (Terumo electronic sphygmomanometer H55, Terumo Co., Ltd., Tokyo, Japan) was wrapped around the upper left arm of the participant.

The length of the forearm between the styloid process of the ulna and the head of the radius was measured, and a point $30 \%$ proximal to the elbow was marked with a marker pen and the circumference at this point was measured on both arms using a tape measure. Similarly, the upper arm length was measured between the lateral epicondyle of the humerus and the acromial process of the scapula, and a point $60 \%$ distal to the acromial process was marked with a marker pen, and then the circumference was measured at the marked point, for both arms. The measurements were carried out before, immediately after, and 15 minutes after the piano performance while the participant stood with the elbow extended and relaxed and the forearm supinated. The coefficient of variation $(\mathrm{CV})$ for this measurement from test to retest was $0.4 \%$ for the forearm and $0.6 \%$ for the upper arm.

Handgrip strength of the left and right arms before, immediately after, and 15 min after the piano performance was measured using a factory-calibrated hand dynamometer (TKK 5401, Takei Co., Ltd., Tokyo, Japan). The participant was 


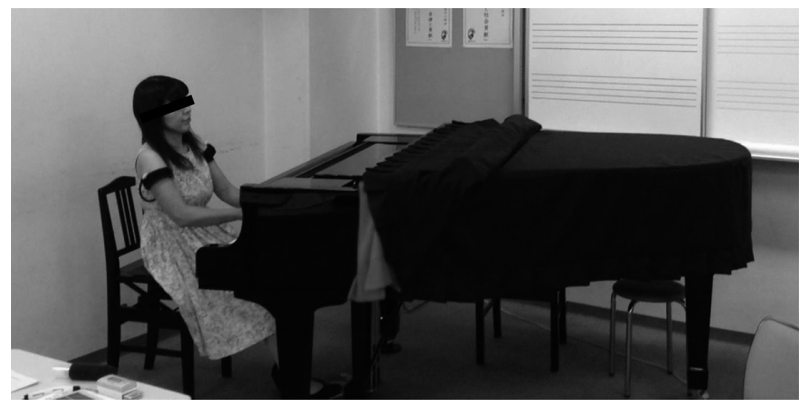

Fig. 1. Piano performance with blood flow restriction was performed while wearing pressure belts at the most proximal region of both arms.

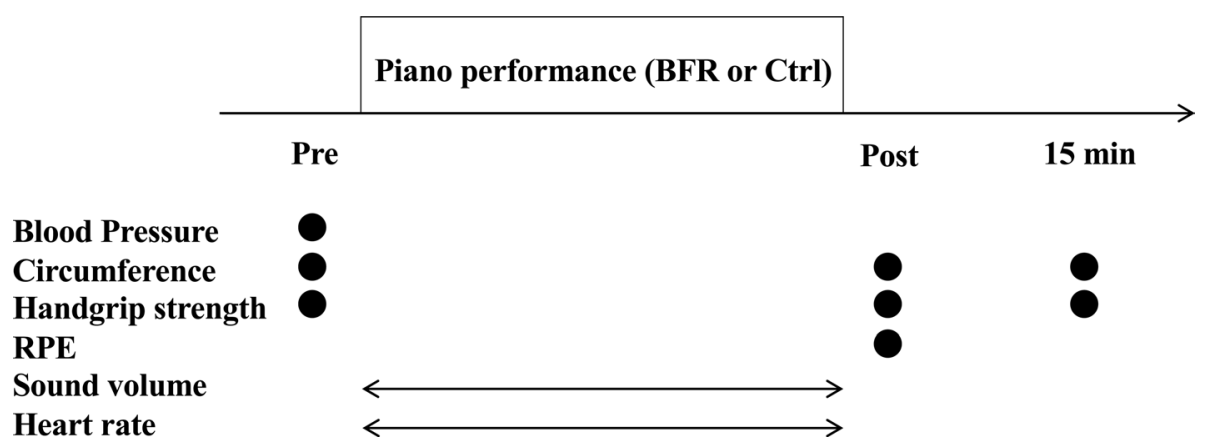

Fig. 2. Experimental timeline-points at which measurements were taken.

-, points of each measurement. $\leftrightarrow$, continuous measurement during piano performance. BFR, blood flow-restricted;

RPE, ratings perceived exertion.

instructed to maintain an upright standing position with arms by her sides, holding the dynamometer with the elbow extended at $180^{\circ}$ without squeezing the arms against the body. The dynamometer's handle was set to a size the participant could comfortably hold and squeeze. The participant was instructed to attain maximal contraction and maintain it for $1-2$ sec. Two trials were performed, and the better of the two trials was used for analysis. The $\mathrm{CV}$ for this measurement from test to retest was $2.2 \%$.

During the experimental session, the sound volume during each piano performance was recorded continuously with a sound level meter (Sound Level Meter MK09, METERK Inc.) from an approximate distance and height of $9 \mathrm{~m}$ and $1.5 \mathrm{~m}$, respectively from the grand piano. The volume meter reading (noise level) was approximately $40-50 \mathrm{~dB}$ before each piano performance, and the maximum value of the sound volume obtained during the piano performance was used as a representative value for the analysis.

During the experimental session, the heart rate during each piano performance was recorded continuously with a fitness watch (A360, POLAR JAPAN Co., Ltd., Tokyo, Japan) placed around the participant's left wrist. The maximum value of the heart rate obtained during each piano performance was used as a representative value in the analysis.

Ratings of perceived exertion (RPE), which measures the subjective feelings of exertion and fatigue of the participant using the Borg scale $(6-20)^{22}$, was recorded immediately after the last set of each piano performance.

The impression of the piano performance was recorded after the piano-BFR. Results are presented as means \pm standard deviation (SD). Statistical analysis was performed using a two-way analysis of variance (ANOVA) with repeated measures [trials (Piano-BFR vs. Piano-Ctrl) $\times$ time (pre vs. post vs. $15 \mathrm{~min}$ )]. Post hoc testing was performed using Tukey's test, as appropriate. Percent changes from pre-were also compared between the groups using Tukey's test. Statistical significance was set at $\mathrm{p}<0.05$. Pre/post effect sizes (ESs, Cohen's d) in circumference and handgrip strength were calculated using the following formula: ([post mean - pre mean] / pre $\mathrm{SD} ; \mathrm{d}=0.2$, small effect; $\mathrm{d}=0.5$, moderate effect; and $\mathrm{d}=0.8$, large effect $)^{23}$ )

\section{RESULTS}

In each experiment, there were no differences $(\mathrm{p}>0.05)$ in temperature $\left(21.5 \pm 2.4\right.$ and $\left.20.3 \pm 1.7^{\circ} \mathrm{C}\right)$, humidity $(32.8 \pm 8.9$ and $33.0 \pm 6.1 \%)$, heart rate $(71 \pm 10$ and $77 \pm 16 \mathrm{BPM})$, systolic $(126 \pm 5$ and $123 \pm 5 \mathrm{mmHg})$ and diastolic blood pressures $(86 \pm 6$ and $81 \pm 6 \mathrm{mmHg}$ ) between the Piano-BFR and the Piano-Ctrl conditions. 
During the piano performance sessions, the heart rate increased to a similar level $(p<0.01)$ in both the Piano-BFR and Piano-Ctrl $(116 \pm 6$ and $123 \pm 10$ BPM). The volume of the sound also increased $(p<0.01)$ to similar levels during the exercise sessions in both the Piano-BFR and Piano-Ctrl $(90 \pm 2$ and $91 \pm 1$ BPM).

Immediately after the piano performance, the forearm circumference showed greater changes $(\mathrm{p}<0.01)$ with the PianoBFR (left 3.6\% and right 5.4\%) than that with the Piano-Ctrl (left $0.4 \%$ and right $0.0 \%$ ) (Table 1). Immediately after the piano performance, the upper arm circumference showed greater changes $(\mathrm{p}<0.01)$ with the Piano-BFR $($ left $2.0 \%$ and right $3.4 \%$ ) than that with the Piano-Ctrl (left $-0.3 \%$ and right $-0.5 \%$ ) (Table 1). All the ESs for the differences in circumference between pre- and post-piano performance were larger for the Piano-BFR (0.8-5.6) than those for the Piano-Ctrl (0.0-0.4). Immediately after the piano performance, handgrip strength for the right arm showed greater changes $(\mathrm{p}<0.01)$ with the Piano-BFR $(-13.4 \%)$ than that with the Piano-Ctrl $(1.3 \%)$, but no changes $(\mathrm{p}>0.05)$ were observed for the Piano-BFR $(-4.1 \%)$ and Piano-Ctrl $(-1.2 \%)$ in the left arm (Table 1). ESs for the differences in handgrip strength between the pre- and post-piano performance were larger for the Piano-BFR (4.7) than those for the Piano-Ctrl (0.2). Immediately after the piano performance, the RPE for the right arm was higher $(\mathrm{p}<0.01)$ in the Piano-BFR $(15.7 \pm 1.4)$ than that in the Piano-Ctrl $(9.5 \pm$ 2.6), but there were no differences $(\mathrm{p}>0.05)$ between the Piano-BFR (12.7 \pm 1.2$)$ and Piano-Ctrl (12.3 \pm 1.9$)$ in the left arm.

\section{DISCUSSION}

We hypothesized that the method of using BFR piano performance as well as BFR resistance exercise may have a significant effect on the neuromuscular mechanism, and we examined the upper limbs of a patient with disabilities who had local neurological symptoms. This study is a preliminary experiment carried out to investigate the acute effects of Piano-BFR on skeletal muscles.

Acute cell swelling has been shown to stimulate protein synthesis and suppress proteolysis ${ }^{24,25)}$. A previous study showed that increased leg circumference, an index of muscle swelling, was more pronounced in BFR conditions than in non-BFR conditions immediately after low-intensity knee extension exercise ${ }^{16)}$. Interestingly, following a single session of low-intensity BFR bench press exercise, acute changes in muscle size were observed in both the blood flow-restricted triceps muscle as well as the blood flow non-restricted chest muscle. The muscle cross-sectional area of both the triceps and chest muscles increased following BFR bench press training ${ }^{26}$. Furthermore, it appears that BFR training-induced muscle cell swelling may contribute significantly to the anabolic benefits of $\mathrm{BFR}^{7,25)}$. In this study, the upper arm circumference increased by 2.0-3.4\% immediately after the piano performance for Piano-BFR (Table 1). In previous studies on BFR resistance exercise, the upper arm circumference increased by 3.5\% (75 repetitions / 4 sets [unpublished data ${ }^{17}$ ]) and $6.4 \%$ (75 repetitions / 4 sets [unpublished data ${ }^{18}$ ]) immediately after the arm curl exercise, suggesting that the circumference changes in this study tended to be lower than that in previous studies. The resistance exercise in the previous study was a single-joint exercise and activated one or two agonist muscles, whereas this study employs a multiple-joint exercise that activates various agonist muscles required for playing the piano. Therefore, the involvement of each muscle during physical activity was vastly different between the resistance exercise and piano performance. Furthermore, the circumference of the forearms showed a larger increase compared to that of the upper arms, and the forearm was directly involved in the movement required when playing the piano. Taken together, blood flow-restricted training effective for rehabilitation of a pianist with residual neurological symptoms in the upper limbs. Furthermore, there is a high possibility that the effect of improvement in the neuromuscular mechanism (muscle hypertrophy and increase in muscle strength, etc.) in the forearm may be larger than that of the upper arms for piano performance.

Compared to the Piano-Ctrl, the Piano-BFR induced greater muscle fatigue in handgrip strength and muscle swelling in the circumference, suggesting that the combination of blood flow restriction and piano performance had a very large effect on

Table 1. Changes in forearm, upper arm circumference, and maximum voluntary contraction (MVC) of the piano performance at pre, immediately post (post), and $15 \mathrm{~min}$ post $(15 \mathrm{~min}$ ) piano performance

\begin{tabular}{|c|c|c|c|c|c|c|c|}
\hline & & \multicolumn{3}{|c|}{ BFR } & \multicolumn{3}{|c|}{$\mathrm{Ctrl}$} \\
\hline & & Pre & Post & $15 \mathrm{~min}$ & Pre & Post & $15 \min$ \\
\hline \multicolumn{8}{|c|}{ Circumference, $\mathrm{cm}$} \\
\hline \multirow[t]{2}{*}{ Forearm } & Left & $21.9(0.2)$ & $22.7(0.3)^{* *}$,\# & $22.1(0.4)^{\#}$ & $21.9(0.2)$ & $21.9(0.2)$ & $21.8(0.2)$ \\
\hline & Right & $22.1(0.2)$ & $23.3(0.4)^{* *, \# \#}$ & $22.7(0.4)^{* *}, \# \#$ & $22.3(0.2)$ & $22.3(0.2)$ & $22.2(0.3)$ \\
\hline \multirow[t]{2}{*}{ Upper arm } & Left & $25.5(0.6)$ & $26.0(0.7)^{* *}, \# \#$ & $25.5(0.5)$ & $25.6(0.6)$ & $25.6(0.6)$ & $25.6(0.7)$ \\
\hline & Right & $25.2(0.4)$ & $26.1(0.5)^{* *}, \# \#$ & $25.3(0.6)$ & $25.3(0.7)$ & $25.2(0.6)$ & $25.4(0.6)$ \\
\hline \multicolumn{8}{|c|}{ Handgrip strength, $\mathrm{kg}$} \\
\hline \multirow[t]{2}{*}{ MVC } & Left & $33.7(2.0)$ & $32.3(1.6)$ & $32.2(2.0)$ & $33.3(1.7)$ & $32.9(2.1)$ & $32.2(1.0)$ \\
\hline & Right & $35.6(1.0)$ & $30.8(2.3)^{* *, \# \#}$ & $32.9(1.4)$ & $34.0(1.7)$ & $34.4(1.3)$ & $33.5(1.2)$ \\
\hline
\end{tabular}

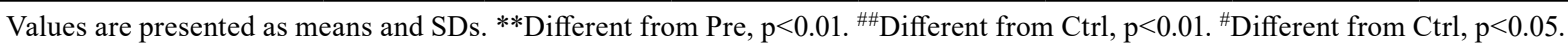


the neuromuscular mechanisms involved. In addition, the remarkable increase in circumferences and RPE and the remarkable decrease in handgrip strength were observed in the right arm compared to that observed in the left arm (Table 1). In this study, the left hand required continuous smooth movements, while the right hand required intermittent movements with multiple fingers at the same time in the "Chopin Revolution". This suggests that during piano performance the activity of the left and right arms was very different. Since musical instrument performances often require asymmetrical physical movements on the left and right sides, it can be speculated that the effects on the left and right arms differ according to the muscle activity in each arm. This laterality is unlikely to occur in resistance exercises and is the cause of the difference observed in the effects of resistance exercise and musical performance. Therefore, further studies are needed to understand the differences between these various exercises and activities.

In this study, the piano performance time was approximately $3 \mathrm{~min}$, and it can be speculated that this was a time setting similar to the resistance exercise (total exercise time of 4 sets) often used in previous studies ${ }^{5,17,18)}$. Additionally, since previous studies reported that rest interval between physical performance with BFR reduces muscle fatigue ${ }^{18,20)}$, BFR condition in this study was an environment that could cause sufficient fatigue compared to the previous studies. As stated earlier, however, the piano performances consisted of physical exercises that activated various upper limb muscles, suggesting that the changes in the circumference (muscle swelling) and maximum strength (muscle fatigue) in the piano performance were smaller than those in the resistance exercises of previous studies that focused on local sites. On the other hand, the heart rate measured during the piano performance was the same as that measured during BFR walking exercise (aerobic exercise) ${ }^{10)}$ regardless of the presence or absence of blood flow restriction, so it may be expected that the Piano-BFR has a beneficial effect similar to aerobic and resistance exercises.

An interesting finding in this study was that the participant reported that "immediately after the Piano-BFR performance was completed and cuff belts were released, the nerves at the fingertips became clear". Therefore, the Piano-BFR condition can be expected to maintain or improve muscle strength by playing the piano within a shorter time than expected in the Piano-Ctrl condition, but the nerve transmission speed may be further improved as a rehabilitation outcome measure of the neuromuscular mechanism for pianists who have residual local neurological symptoms.

This study has some limitations. First, the pressure for the resistance exercise with blood flow restriction was applied at $130-170 \mathrm{mmHg}$ for the participant, and because of this arbitrary pressure, there were large differences compared to results from other studies ${ }^{17,18,27)}$. Second, following resistance exercise the endocrine system is also associated with muscle fatigue $^{13,14,27)}$, but it is uncertain how this system is affected. Third, we considered that musical characteristics had an effect in neuromuscular mechanism, but the effect of the laterality of the patient's neurological symptoms cannot be completely denied. Further studies are needed to understand the relationship between Piano-BFR, neuromuscular mechanisms, and/or the effects of training.

In conclusion, blood flow-restricted training, known as KAATSU training, is effective for rehabilitation of a pianist with residual neurological symptoms in the upper limbs. Furthermore, there is a high possibility that the effect of improvement in the neuromuscular mechanism (muscle hypertrophy and increase in muscle strength, etc.) in the forearm may be larger than that of the upper arms for piano performance. This study is a preliminary experiment, and it is necessary to conduct further clinical studies in the future to confirm the acute and chronic effects from the viewpoint of resistance exercise as well as aerobic exercise.

\section{Funding}

This study was supported in part by JSPS KAKENHI, Grant Number 18K10906 (to T.Y.).

\section{Conflicts of interest}

Toshiaki Nakajima belongs to a donation course supported by KAATSU JAPAN Co., Ltd., but KAATSU JAPAN Co., LTD had no role in the design and analysis of the data. The other authors have declared that no competing interests exist.

\section{REFERENCES}

1) Portal Site of Official Statistics of Japan: 1 Road traffic accidents [Survey date 2019]. https://www.e-stat.go.jp/en/stat-search/files?page=1\&layout=datalist\&to ukei $=00130002 \&$ tstat $=000001027457 \&$ cycle $=7 \&$ year $=20190 \&$ month $=0$ (Accessed Mar. 4,2021$)$

2) Maeda T, Katayama Y, Yoshino A: Current status and issues of the sequelae following the traffic accident. Jpn J Acute Med, 2018, 42: 715-721 (in Japanese).

3) American College of Sports Medicine: American College of Sports Medicine position stand. Progression models in resistance training for healthy adults. Med Sci Sports Exerc, 2009, 41: 687-708. [Medline] [CrossRef]

4) Ozaki H, Yasuda T, Ogasawara R, et al.: Effects of high-intensity and blood flow-restricted low-intensity resistance training on carotid arterial compliance: role of blood pressure during training sessions. Eur J Appl Physiol, 2013, 113: 167-174. [Medline] [CrossRef]

5) Yasuda T, Fukumura K, Fukuda T, et al.: Muscle size and arterial stiffness after blood flow-restricted low-intensity resistance training in older adults. Scand J Med Sci Sports, 2014, 24: 799-806. [Medline] [CrossRef]

6) Nakajima T, Yasuda T, Sato Y, et al.: Effects of exercise and anti-aging. Anti-Aging Med, 2011, 8: 92-102. [CrossRef]

7) Yasuda T, Loenneke JP, Thiebaud RS, et al.: Effects of blood flow restricted low-intensity concentric or eccentric training on muscle size and strength. PLoS 
One, 2012, 7: e52843. [Medline] [CrossRef]

8) Yasuda T, Fukumura K, Uchida Y, et al.: Effects of low-load, elastic band resistance training combined with blood flow restriction on muscle size and arterial stiffness in older adults. J Gerontol A Biol Sci Med Sci, 2015, 70: 950-958. [Medline] [CrossRef]

9) Yasuda T, Oosumi S, Sugimoto S, et al.: Effect of KAATSU training on thigh muscle size and safety for a patient with knee meniscectomy over 3 years. Int J KAATSU Train Res, 2017, 13: 11-14. [CrossRef]

10) Abe T, Kearns CF, Sato Y: Muscle size and strength are increased following walk training with restricted venous blood flow from the leg muscle, Kaatsu-walk training. J Appl Physiol 1985, 2006, 100: 1460-1466. [Medline]

11) Natsume T, Ozaki H, Saito AI, et al.: Effects of electrostimulation with blood flow restriction on muscle size and strength. Med Sci Sports Exerc, 2015, 47: 2621-2627. [Medline] [CrossRef]

12) Nakajima $T$, Kurano M, Iida $H$, et al.: Use and safety of KAATSU training: results of a national survey. Int J KAATSU Train Res, 2006, 2: 5-13. [CrossRef]

13) Yasuda T, Meguro M, Sato Y, et al.: Use and safety of KAATSU training: results of a national survey in 2016. Int J KAATSU Train Res, 2017, 13: 1-9. [CrossRef]

14) Loenneke JP, Fahs CA, Rossow LM, et al.: The anabolic benefits of venous blood flow restriction training may be induced by muscle cell swelling. Med Hypotheses, 2012, 78: 151-154. [Medline] [CrossRef]

15) Pearson SJ, Hussain SR: A review on the mechanisms of blood-flow restriction resistance training-induced muscle hypertrophy. Sports Med, 2015, 45: 187200. [Medline] [CrossRef]

16) Fry CS, Glynn EL, Drummond MJ, et al.: Blood flow restriction exercise stimulates mTORC1 signaling and muscle protein synthesis in older men. J Appl Physiol 1985, 2010, 108: 1199-1209. [Medline]

17) Yasuda T, Brechue WF, Fujita T, et al.: Muscle activation during low-intensity muscle contractions with varying levels of external limb compression. J Sports Sci Med, 2008, 7: 467-474. [Medline]

18) Yasuda T, Brechue WF, Fujita T, et al.: Muscle activation during low-intensity muscle contractions with restricted blood flow. J Sports Sci, 2009, 27: 479-489. [Medline] [CrossRef]

19) Sanchez-Delgado G, Cadenas-Sanchez C, Mora-Gonzalez J, et al.: Assessment of handgrip strength in preschool children aged 3 to 5 years. J Hand Surg Eur Vol, 2015, 40: 966-972. [Medline] [CrossRef]

20) Yasuda T, Fukumura K, Iida H, et al.: Effect of low-load resistance exercise with and without blood flow restriction to volitional fatigue on muscle swelling Eur J Appl Physiol, 2015, 115: 919-926. [Medline] [CrossRef]

21) Yasuda T, Abe T, Brechue WF, et al.: Venous blood gas and metabolite response to low-intensity muscle contractions with external limb compression. Metabolism, 2010, 59: 1510-1519. [Medline] [CrossRef]

22) Borg GA: Psychophysical bases of perceived exertion. Med Sci Sports Exerc, 1982, 14: 377-381. [Medline] [CrossRef]

23) Cohen J: Statistical power analysis for the behavioral sciences, 2nd ed. Hillsdale: Lawrence Erlbaum Associates, 1988, pp 19-39.

24) Berneis K, Ninnis R, Häussinger D, et al.: Effects of hyper- and hypoosmolality on whole body protein and glucose kinetics in humans. Am J Physiol, 1999, 276: E188-E195. [Medline]

25) Häussinger D, Roth E, Lang F, et al.: Cellular hydration state: an important determinant of protein catabolism in health and disease. Lancet, 1993, 341: 1330-1332. [Medline] [CrossRef]

26) Yasuda T, Fujita S, Ogasawara R, et al.: Effects of low-intensity bench press training with restricted arm muscle blood flow on chest muscle hypertrophy: a pilot study. Clin Physiol Funct Imaging, 2010, 30: 338-343. [Medline] [CrossRef]

27) Loenneke JP, Fahs CA, Rossow LM, et al.: Effects of cuff width on arterial occlusion: implications for blood flow restricted exercise. Eur J Appl Physiol, 2012, 112: 2903-2912. [Medline] [CrossRef] 